\title{
GLOBAL ROBUST STABILITY OF TIME-DELAY SYSTEMS WITH DISCONTINUOUS ACTIVATION FUNCTIONS UNDER POLYTOPIC PARAMETER UNCERTAINTIES
}

\author{
Zengyun Wang, Lihong Huang, Yi Zuo, and Lingling Zhang
}

\begin{abstract}
This paper concerns the problem of global robust stability of a time-delay discontinuous system with a positive-defined connection matrix under polytopic-type uncertainty. In order to give the stability condition, we firstly address the existence of solution and equilibrium point based on the properties of $M$-matrix, Lyapunov-like approach and the theories of differential equations with discontinuous right-hand side as introduced by Filippov. Second, we give the delay-independent and delay-dependent stability condition in terms of linear matrix inequalities (LMIs), and based on Lyapunov function and the properties of the convex sets. One numerical example demonstrate the validity of the proposed criteria.
\end{abstract}

\section{Introduction}

Time delay often as a source of instability and oscillations are frequently encountered in various areas, including engineering, biology and economics [17]. Hence, the stability of delayed system has received notable attention in the past few years $[10,12,19,16]$. However, all above papers are considering the continuous system. As [15] pointed out, neural networks with discontinuous activation functions are important and frequently arise in practice when dealing with dynamical systems possessing high-slope nonlinear elements. For this reason, considerable effort has been devoted to analyzing the dynamical behavior of neural networks with discontinuous activation function $[7,8,5,18]$.

Received October 24, 2008.

2000 Mathematics Subject Classification. 93D09, 34D20, 34D23.

Key words and phrases. global robust stability, delayed neural network, delay-independent condition, delay-dependent condition, linear matrix inequality, discontinuous neuron activation.

This work was supported by National Natural Science Foundation of China (10771055, 60775047, 60835004), Major Subject of Ministry of Education of China (706043), Graduate Innovation Foundation of Hunan Province (CX2009B065), Foundation of China Scholarship Council ([2008]3019), Foundation of NSERC Canada, the Specialized Research Fund for the Doctoral Program of Higher Education (20050532023) and National High Technology Research and Development Program of China (863 Program: 2007AA04Z244, 2008AA04Z214). 
The model discussed in this paper is an extension of the model proposed in $[9,11]$, and it is widely applied to solve various optimization problems such as the linear variational inequality problem that contains linear convex quadratic programming and linear complementary problem as special cases $[2,13,6]$. To the best of our knowledge, there is little results towards the general neural networks with discontinuous neuron activation, which the self-connection matrix is not a real diagonal matrix, but a positive-defined matrix. In this paper, based on Filippov theories [4] and the properties of $M$-matrix [12], we discussed the existence of solution and equilibrium for the general time delay systems.

In practical implementation of neural networks, uncertainties are inevitable in neural networks because of the existence of modelling errors and external disturbance. In the past few years, the robust stability of uncertain systems with time delay has received the considerable attention and many papers have focus on time delay systems with polytopic-type uncertainty [10, 14]. However there is little result considers the discontinuous system. In this paper, we consider the system with discontinuous activation function. We address the robust stability condition based on Lyapunov function, properties of convex set and linear matrix inequality (LMI) technology. The method presented in $[3,19]$ is also employed to derive the delay-dependent stability condition for the time-delay discontinuous systems.

The paper is organized as follows. Section 2 presents some assumptions and preliminaries which are used in the following sections. Section 3 deals with the existence of solution for the general time delay systems with discontinuous activation and gives the condition of the existence and uniqueness of the equilibrium. The main results, which guarantee the global robust stability of the polytopic-type uncertainty system, are presented in Section 4 . Section 5 illustrates the results on an example borrowed the literature. Finally some concluding remarks end the paper.

\section{Neural network model}

Consider a class of recurrent neural networks (RNNS) which was proposed in $[9,11]$ and described in vector form by:

$$
\frac{\mathrm{d} y(t)}{\mathrm{d} t}=-D y(t)+g(W y(t)+I)
$$

where $y(t)=\left(y_{1}(t), y_{2}(t), \ldots, y_{n}(t)\right)^{\mathrm{T}}$ is the state vector of the neural network; $D=\operatorname{diag}\left\{d_{1}, d_{2}, \ldots, d_{n}\right\}$ denotes an the real diagonal matrix with $d_{i}>$ $0 ; W=\left[w_{i j}\right]_{n \times n}$ is the constant connection weight matrix, and $g(y(t))=$ $\left(g_{1}\left(y_{1}(t)\right), g_{2}\left(y_{2}(t)\right), \ldots, g_{n}\left(y_{n}(t)\right)\right)^{\mathrm{T}}$ is a vector valued nonlinear activation function from $\mathbb{R}^{n}$ to $\mathbb{R}^{n} ; I=\left(I_{1}, I_{2}, \ldots, I_{n}\right)^{\mathrm{T}} \in \mathbb{R}^{n}$ means the vector of constant neuron inputs. 
Here we consider an extension of system (2.1) with time delay, which can be described by the following differential equations:

$$
\frac{\mathrm{d} y(t)}{\mathrm{d} t}=-D y(t)+A g(W y(t)+I)+B g(W y(t-\tau)+I) .
$$

If $W$ is a nonsingular matrix, by using $x(t)=W y(t)+I$, the model (2.1) is transformed to

$$
\frac{\mathrm{d} x(t)}{\mathrm{d} t}=-W D W^{-1} x(t)+W A g(x(t))+W B g(x(t-\tau))+W D W^{-1} I,
$$

and in most cases $W D W^{-1}$ is not a real diagonal matrix, but a positive defined matrix. In the following discussion of our paper, we make two assumptions.

$\mathbf{A}(\mathbf{1}): A D=D A, B D=D B$, obviously, $A D^{-1}=D^{-1} A, B D^{-1}=D^{-1} B$.

$\mathbf{A}(2)$ : The discontinuous activation function $g_{i} \in \mathscr{G}$ for any $i=1,2, \ldots, n$, where $\mathscr{G}$ denotes the class of functions from $\mathbb{R}$ to $\mathbb{R}$ which are monotone nondecreasing and continuous except on a countable set of isolate points $\left\{\rho_{k}^{i}\right\}$, where the right and left limits $g_{i}^{+}\left(\rho_{k}^{i}\right)$ and $g_{i}^{-}\left(\rho_{k}^{i}\right)$ satisfy $g_{i}^{+}\left(\rho_{k}^{i}\right)>g_{i}^{-}\left(\rho_{k}^{i}\right)$. Moreover, in every compact set of $\mathbb{R}, g_{i}$ has only finite discontinuous points.

We note that if $g$ satisfies $\mathrm{A}(2)$, then any $g_{i}, i=1,2, \ldots, n$, possesses only isolated jump discontinuities where $g_{i}$ is not necessarily defined. Hence for all $x \in \mathbb{R}^{n}$, we have

(See [7, Definition 4]).

$$
\begin{aligned}
K[g(\xi)] & =\left(K\left[g_{1}(\xi)\right], \ldots, K\left[g_{n}(\xi)\right]\right)^{\mathrm{T}} \\
& =\left(\left[g_{1}^{-}(\xi), g_{1}^{+}(\xi)\right], \ldots,\left[g_{n}^{-}(\xi), g_{n}^{+}(\xi)\right]\right)^{\mathrm{T}}
\end{aligned}
$$

In practice, the weight coefficients of the neurons depend on certain resistance and capacitance values, which are subject to uncertainties. Therefore in this paper, we consider the system with constant uncertain real parameters that can be described by

$$
\frac{\mathrm{d} x(t)}{\mathrm{d} t}=-W D_{\theta} W^{-1} x(t)+W A_{v} g(x(t))+W B_{\omega} g(x(t-\tau))+W D_{\theta} W^{-1} I
$$

For convenience of discussion in the later context, we denote this neural delay differential equation as follows:

$$
\frac{\mathrm{d} x(t)}{\mathrm{d} t}=-D_{\theta} x(t)+A_{v} g(x(t))+B_{\omega} g(x(t-\tau))+D_{\theta} I,
$$

where the uncertain real parameters and the system matrix $D_{\theta}, A_{v}$ and $B_{\omega}$ are represented as

$$
D_{\theta}=D+\sum_{i=1}^{k} \theta_{i} D_{i}, \quad A_{v}=A+\sum_{j=1}^{p} v_{j} A_{j}, \quad B_{\omega}=B+\sum_{l=1}^{m} \omega_{l} B_{l},
$$

where $D, D_{i}, A, A_{j}, B, B_{l}$ are known fixed matrixes, and $\theta=\left(\theta_{1}, \theta_{2}, \ldots, \theta_{k}\right)^{\mathrm{T}} \in$ $\mathbb{R}^{k}, v=\left(v_{1}, v_{2}, \ldots, v_{p}\right)^{\mathrm{T}} \in \mathbb{R}^{p}$ and $\omega=\left(\omega_{1}, \omega_{2}, \ldots, \omega_{m}\right)^{\mathrm{T}} \in \mathbb{R}^{m}$. 
We assume that lower and upper bounds are available for the parameter $\theta, v, \omega$ respectively. Specifically, each parameter $\theta_{i}, v_{j}$ and $\omega_{l}$ ranges between known external values $\underline{\theta}_{i}$ and $\bar{\theta}_{i}, \underline{v}_{j}$ and $\bar{v}_{j}, \underline{\omega}_{l}$ and $\bar{\omega}_{l}$, equivalently, $\theta_{i} \in\left[\underline{\theta}_{i}, \overline{\theta_{i}}\right]$, $v_{j} \in\left[\underline{v}_{j}, \bar{v}_{j}\right]$ and $\omega_{l} \in\left[\underline{\omega}_{l}, \bar{\omega}_{l}\right]$. In order to discuss our problem, we set the following sets:

$$
\begin{aligned}
& \Theta:=\left\{\bar{D}_{\theta}=D+\sum_{i=1}^{k} \theta_{i}^{\prime} D_{i}: \theta_{i}^{\prime} \in\left\{\underline{\theta}_{i}, \overline{\theta_{i}}\right\}\right\}, \\
& \Upsilon:=\left\{\bar{A}_{v}=A+\sum_{j=1}^{p} v_{j}^{\prime} A_{j}: v_{j}^{\prime} \in\left\{\underline{v}_{j}, \overline{v_{j}}\right\}\right\}, \\
& \Omega:=\left\{\bar{B}_{\omega}=B+\sum_{l=l}^{m} \omega_{l}^{\prime} B_{l}: \omega_{l}^{\prime} \in\left\{\underline{\omega}_{l}, \overline{\omega_{l}}\right\}\right\},
\end{aligned}
$$

then we see $\bar{D}_{\theta}, \bar{A}_{v}, \bar{B}_{\omega}$ denotes $2^{k}, 2^{p}, 2^{m}$ vertices of the sets $\Theta, \Upsilon, \Omega$ respectively. The nominal system can be described as

$$
\dot{x}(t)=-D x(t)+A g(t)+B g(t-\tau)+D I .
$$

\section{Existence of solution and equilibrium}

All the definitions and properties concerning our stability analysis for the nominal system can be found in ([4]). In this section, we firstly introduce the global robust stability conception about the uncertain delayed neural network system. After that we discuss the existence and uniqueness equilibrium of the system.

Definition 3.1. The network model given by (2.5) with the parameter are valued in the parameter boxes is globally robust stable if the equilibrium point $x^{\star}$ of the model is globally asymptotically stable and the unique output equilibrium point $\gamma^{\star}$ corresponding to the equilibrium point $x^{\star}$ is globally output convergent in measure for all $\theta \in \Theta, v \in \Upsilon, \omega \in \Omega$.

The existence of solution can be obtained with the similar method in ([4]), one also gets the following properties for the system which the matrix $D$ is a positive defined matrix.

Property 3.1. If $\mathrm{A}(2)$ is satisfied, the IVP of system (2.6) has at least a maximal solution $[x, \gamma]$ on $[0, T)$ for some $T \in[0,+\infty)$.

Based on Property 3.1, the IVP of (2.6) has a solution in some time $[0, T)$ where $T \in[0,+\infty)$ such that $[0, T)$ is the maximal right existence interval of the solution $x(t)$.

By virtue of the continuation theorem of differential equations with discontinuous right-hand side ([4, p.78, Th.2]), we can conclude that:

Property 3.2. Suppose A(2) are satisfied. Then the IVP of system (2.6) has a bounded absolutely continuous solution $x(t)$ for $t \in[0,+\infty)$. 
Base on the above properties, we introduce the existence and uniqueness of equilibrium point and the corresponding output equilibrium point of system (2.3). We firstly present the following definition and equilibrium theorem which will be used in the proof of Theorem 3.4.

Definition $3.2([1])$. Suppose $K$ is a convex subset of $\mathbb{R}^{n}$. The tangent cone $T_{K}(x)$ to $K$ at $x \in K$ is defined as

$$
T_{K}(x)=\bigcup_{h>0} \frac{K-x}{h},
$$

where $\bar{U}$ is the closure of the union set.

Theorem 3.3 ([1]). Let us consider a compact convex subset $K \in \mathbb{R}^{n}$ with nonempty interior and an upper semicontinuous set-valued map $F$ from $[0,1] \times$ $K$ to $\mathbb{R}^{n}$, with nonempty closed convex values. Suppose the set-valued map $x \rightarrow F(0, x)$ satisfies the tangential condition

$$
\forall x \in \partial K, \quad F(0, x) \cap T_{K}(x) \neq \emptyset
$$

and

$$
\forall x \in \partial K, \forall \lambda \in[0,1], 0 \notin F(0, x),
$$

then there exists $\bar{x} \in K$ such that $0 \in F(1, \bar{x})$.

Theorem 3.4 (existence and uniqueness of equilibrium point). Suppose that $A(1)$ and $A(2)$ are satisfied, $-W A$ and $-W B$ are nonsingular $M$-matrix, then system (2.3) has a unique equilibrium point $x^{*}$ and a unique corresponding output equilibrium point $\eta^{*}$.

Proof. Let $x^{*}=\left(x_{1}^{*}, x_{2}^{*}, \ldots, x_{n}^{*}\right) \in \mathbb{R}^{n}$ denote an equilibrium point of the neural network model (2.3). From the definition of equilibrium, $x^{*}$ satisfies the inclusion $\dot{x}(t) \in-D x(t)+A \gamma(t)+B \gamma(t-\tau)+I$. Since $-W A$ and $-W B$ are nonsingular $M$-matrix, $-W(A+B)$ is a nonsingular $M$-matrix, and we get

$$
0 \in-x^{*}+W D^{-1} A K\left[g\left(x^{*}\right)\right]+W D^{-1} B K\left[g\left(x^{*}\right)\right]+I .
$$

Because $-W(A+B)$ is $M$-matrix, there exists constant $r_{i}>0$ such that

$$
-r_{i}\left(\sum_{j=1}^{n} w_{i j}\left(a_{j i}+b_{j i}\right)\right)>\sum_{k=1, k \neq i}^{n} r_{k}\left|\sum_{j=1}^{n} w_{i j}\left(a_{j k}+b_{j k}\right)\right| .
$$

Let

$$
0 \in \varphi(x)=-x+W D^{-1} A K[g(x)]+W D^{-1} B K[g(x(t-\tau)]+I .
$$

Obviously, the solution of inclusion (3.2) is the equilibrium point of model (2.3). Defining a family of homotopic multi-valued maps as follows:

$$
\begin{aligned}
\Phi(x, \lambda) & =\lambda \varphi(x)+(1-\lambda)(-x) \\
& =-x+\lambda W D^{-1} A K[g(x)]+\lambda W D^{-1} B K[g(x(t-\tau)]+\lambda I,
\end{aligned}
$$


where $\lambda \in[0,1]$. Using (3.3), for a fixed point $x \in \mathbb{R}^{n}$ and any

$$
p=\left(p_{1}, p_{2}, \ldots, p_{n}\right)^{\mathrm{T}} \in \Phi(x, \lambda),
$$

there exists some $\eta=\left(\eta_{1}, \eta_{2}, \ldots, \eta_{n}\right)^{\mathrm{T}} \in K[g(x)]$, such that

$$
p=-x+\lambda W D^{-1} A \eta+\lambda W D^{-1} B \eta+\lambda I .
$$

According to the monotonicity of $g_{i}\left(x_{i}\right)$, we define a vector

$$
v=\left(v_{1}, v_{2}, \ldots, v_{n}\right)^{\mathrm{T}}
$$

with

$$
v_{i}=\left\{\begin{array}{l}
\text { any value, } \quad \text { if } x_{i}=\eta_{i}=0, \\
r_{i} \operatorname{sign}\left(\eta_{i}\right), \quad \text { if } x_{i}=0, \eta_{i} \neq 0, \\
r_{i} \operatorname{sign}\left(x_{i}\right)=r_{i} \operatorname{sign}\left(\eta_{i}\right), \quad \text { if } x_{i} \neq 0, \eta_{i} \neq 0,
\end{array}\right.
$$

where $r_{i}$ satisfies (3.1). It follows that $v_{i} x_{i}=r_{i}\left|x_{i}\right|$ and $v_{i} \eta_{i}=r_{i}\left|\eta_{i}\right|$ for any $i \in\{1,2, \ldots, n\}$. Using inequality (3.1), we have

$$
\begin{aligned}
v^{\mathrm{T}} p & =v^{\mathrm{T}}\left[-x+\lambda W D^{-1}(A+B) \eta+\lambda I\right] \\
& =v^{\mathrm{T}}\left[-x+\lambda W(A+B) D^{-1} \eta+\lambda I\right] \\
& \leq \sum_{i=1}^{n}\left\{-r_{i}\left|x_{i}\right|+\lambda r_{i}\left[\sum_{j=1}^{n} w_{i j}\left(a_{j i}+b_{j i}\right) d_{i}^{-1}\left|\eta_{i}\right|\right]\right. \\
& \left.\quad+\lambda \sum_{k=1, k \neq i}^{n} r_{k}\left|\sum_{j=1}^{n} w_{i j}\left(a_{j k}+b_{j k}\right)\right| d_{i}^{-1}\left|\eta_{i}\right|\right\}+\lambda \sum_{i=1}^{n} r_{i}\left|I_{i}\right| \\
& \leq-\sum_{i=1}^{n} r_{i}\left|x_{i}\right|+\lambda \sum_{i=1}^{n}\left[r_{i} \sum_{j=1}^{n} w_{i j}\left(a_{j k}+b_{j k}\right)\right. \\
& \left.+\sum_{k=1, k \neq i}^{n}\left|r_{k}\right| \sum_{j=1}^{n} w_{i j}\left(a_{j k}+b_{j k}\right) \mid\right] d_{i}^{-1}\left|\eta_{i}\right|+\lambda \sum_{i=1}^{n} r_{i}\left|I_{i}\right| \\
\leq & -r_{\min }\|x\|_{1}+r_{\max }\|I\|_{1},
\end{aligned}
$$

where $r_{\min }=\min _{1 \leq i \leq n}\left\{r_{i}\right\}, r_{\max }=\max _{1 \leq i \leq n}\left\{r_{i}\right\}$. Let

$$
U\left(R_{0}\right)=\left\{x \mid\|x\|_{1}<R_{0}=\frac{r_{\max }}{r_{\min }}\left(\|I\|_{1}+1\right)\right\} .
$$

Then, it follows from (3.6) that $\|x\|_{1}=R_{0}=\frac{r_{\max }}{r_{\min }}\left(\|I\|_{1}+1\right)$ for any $x \in \partial U\left(R_{0}\right)$, and we have

$$
v^{\mathrm{T}} p \leq-r_{\min } \cdot \frac{r_{\max }}{r_{\min }}\left(\|I\|_{1}+1\right)+r_{\max }\|I\|_{1}<0, \quad \forall \lambda \in[0,1],
$$

that is $p \neq 0$, i.e., $0 \notin \Phi(x, \lambda)$ for any $x \in \partial U\left(R_{0}\right), \lambda \in[0,1]$. Also, as $\lambda=0$, $\Phi(x, 0)=-x$, there is $\Phi(x, 0) \cap T_{U\left(R_{0}\right)}(x)=\{-x\} \notin \emptyset$. By Theorem 3.3, we can conclude that there exist $x^{*} \in U\left(R_{0}\right)$ such that $0 \in \Phi\left(x^{*}, 1\right)=\Phi\left(x^{*}\right)$. 
This implies that system (2.3) has at least one equilibrium point $x^{*}$. From the definition (IVP) of the system, there exists $\eta \in K\left[g\left(x^{*}\right)\right]$ such that

$$
0=-x^{*}+W D^{-1} A \eta^{*}+W D^{-1} B \eta^{*}+I .
$$

Hence, the existence of output equilibrium point $\eta^{*}$ corresponding to $x^{*}$ is also obtained.

In the following we prove the uniqueness equilibrium point. For contradiction, there exist two distinct equilibrium points $x_{1}^{*}$ and $x_{2}^{*}$, which correspond to two output equilibrium $\eta_{1}^{*}$ and $\eta_{2}^{*}$, such that

$$
\begin{aligned}
& -x_{1}^{*}+W D^{-1} A \eta_{1}^{*}+W D^{-1} B \eta_{2}^{*}+I=0, \\
& -x_{2}^{*}+W D^{-1} A \eta_{2}^{*}+W D^{-1} B \eta_{2}^{*}+I=0 .
\end{aligned}
$$

It follows that

$$
-\left(x_{1}^{*}-x_{2}^{*}\right)+W D^{-1}(A+B)\left(\eta_{1}^{*}-\eta_{2}^{*}\right)=0 .
$$

By $\mathrm{A}(2), g_{i}\left(x_{i}\right)$ is nondecreasing functions, so there exists $L=\operatorname{diag}\left(l_{1}, l_{2}, \ldots\right.$, $\left.l_{n}\right)$ such that

$$
\eta_{1}^{*}-\eta_{2}^{*}=L\left(x_{1}^{*}-x_{2}^{*}\right)
$$

Then, (3.8) can be written as

$$
\left(-I+W D^{-1}(A+B) L\right)\left(x_{1}^{*}-x_{2}^{*}\right)=0 .
$$

Since $-W(A+B)$ is $M$-matrix, $D^{-1} L$ is a non-negative diagonal matrix, we have $\operatorname{det}\left(-I+W D^{-1}(A+B) L\right) \neq 0$. This means that $x_{1}^{*}=x_{2}^{*}$, which is a contradiction. Hence system (2.3) has a unique equilibrium point $x^{*}$ and a unique corresponding output equilibrium point $\eta^{*}$.

\section{Global robust stability criterion}

Suppose $x^{\star}$ is an equilibrium point of (2.3), that is, there exists $\gamma^{\star}=$ $\left(\gamma_{1}^{\star}, \ldots, \gamma_{n}^{\star}\right) \in K\left[g\left(x^{\star}\right)\right]$ such that $-D x^{*}+(A+B) \gamma^{*}+I=0$ is satisfied. Let $z(t)=x(t)-x^{\star}$ and $\kappa(t)=\gamma(t)-\gamma^{\star}$. Then $z(t)=\left(z_{1}(t), \ldots, z_{n}(t)\right)^{\mathrm{T}}$ satisfies

$$
\frac{\mathrm{d} z(t)}{\mathrm{dt}}=-D z(t)+A \kappa(t)+B \kappa(t-\tau) \text { for almost all } t,
$$

where $\kappa(t) \in K[f(y(t))], f_{i}\left(z_{i}(t)\right)=g_{i}\left(z_{i}(t)+x_{i}^{*}\right)-\kappa_{i}^{*}, i=1,2, \ldots, n$.

In this part, we will discuss the global robust stability of system (2.5) based on Lyapunov functions and give the stability conditions in terms of linear Matrix inequalities.

First of all, we give the definition of absolute stability of system (2.5).

Definition 4.1. If there exists a Lyapunov function $V_{[z, \kappa]}(t)$ of system $(4.1)$ with a negative definite derivation $\left.\dot{V}_{[z, \kappa]}(t)\right|_{(4.1)} \leq 0$, then we say system of (4.1) is robustly absolutely stable. This also means that system (2.5) is global robust stable about $x^{*}$. 


\subsection{Delay-independent robust stability}

Theorem 4.2. Suppose that $A(1)$ and $A(2)$ are satisfied, if there exist $P=$ $P^{\mathrm{T}}>0, Q=Q^{\mathrm{T}}>0$ and $\wedge=\operatorname{diag}\left(\lambda_{1}, \lambda_{2}, \ldots, \lambda_{n}\right) \geq 0$ such that

$$
\left[\begin{array}{ccc}
-P \bar{D}_{\theta}-\bar{D}_{\theta}^{\mathrm{T}} P & P \bar{A}_{v}-\bar{D}_{\theta} \wedge & P \bar{B}_{\omega} \\
\bar{A}_{v}^{\mathrm{T}} P-\wedge \bar{D}_{\theta}^{\mathrm{T}} & \wedge \bar{A}_{v}+\bar{A}_{v}^{\mathrm{T}} \wedge+Q & \wedge \bar{B}_{\omega} \\
\bar{B}_{\omega}^{\mathrm{T}} P & \bar{B}_{\omega}^{\mathrm{T}} \wedge & -Q
\end{array}\right]<0
$$

holds for any $\bar{D}_{\theta} \in \Theta, \bar{A}_{v} \in \Upsilon$ and $\bar{B}_{\omega} \in \Omega$, then system (2.5) is robust stable.

Proof. Choose a Lyapunov function candidate to be

$$
V_{[z, \kappa]}=z^{\mathrm{T}}(t) P z(t)+2 \sum_{i=1}^{n} \lambda_{i} \int_{0}^{z_{i}(t)} f_{i}(s) \mathrm{d} s+\int_{t-\tau}^{t} \kappa^{\mathrm{T}}(\rho) Q \kappa(\rho) \mathrm{d} \rho,
$$

where $P=P^{\mathrm{T}}>0, Q=Q^{\mathrm{T}}>0$ and $\lambda_{i} \geq 0(i=1,2, \ldots, n)$ are positive constants. Differentiating $V_{[z, \kappa]}$ by the chain role, we have

$$
\begin{aligned}
\dot{V}_{[z, \kappa]}= & 2 z^{\mathrm{T}}(t) P \dot{z}(t)+2 \kappa^{\mathrm{T}}(t) \wedge \dot{z}(t)+\kappa^{\mathrm{T}}(t) Q \kappa(t)-\kappa^{\mathrm{T}}(t-\tau) Q \kappa(t-\tau) \\
= & -2 z^{\mathrm{T}}(t) P D_{\theta} z(t)+2 z^{\mathrm{T}}(t) P A_{v} \kappa(t)+2 z^{\mathrm{T}}(t) P B_{\omega} \kappa(t-\tau) \\
& -2 \kappa^{\mathrm{T}}(t) \wedge D_{\theta} z(t)+2 \kappa^{\mathrm{T}}(t) \wedge \bar{A}_{v} \kappa(t)+2 \kappa^{\mathrm{T}}(t) \wedge B_{\omega} \kappa(t-\tau) \\
& +\kappa^{\mathrm{T}}(t) Q \kappa(t)-\kappa^{\mathrm{T}}(t-\tau) Q \kappa(t-\tau) \\
= & {\left[z^{\mathrm{T}}(t), \kappa^{\mathrm{T}}(t), \kappa^{\mathrm{T}}(t-\tau)\right] \Pi[z(t), \kappa(t), \kappa(t-\tau)]^{\mathrm{T}}, }
\end{aligned}
$$

where

$$
\Pi=\left[\begin{array}{ccc}
-P D_{\theta}-D_{\theta}^{\mathrm{T}} P & P A_{v}-D_{\theta} \wedge & P B_{\omega} \\
A_{v}^{\mathrm{T}} P-\wedge D_{\theta}^{\mathrm{T}} & \wedge A_{v}+A_{v}^{T} \wedge+Q & \wedge B_{\omega} \\
B_{\omega}^{\mathrm{T}} P & B_{\omega}^{\mathrm{T}} \wedge & -Q
\end{array}\right]
$$

From the definition of set $\Theta, \Upsilon$ and $\Omega$, we know $\Theta, \Upsilon$ and $\Omega$ are convex sets. By the convexity of $\Theta, \Upsilon$ and $\Omega$, we obtain that if (4.2) holds for every $\bar{D}_{\theta}, \bar{A}_{v}$ and $\bar{B}_{\omega}$, then we know that $(4.3)$ can be hold.

So we get $\left.\dot{V}_{[z, \kappa]}\right|_{(4.1)}<0$. From the definition of absolute stability of system, we will get system (4.1) is global robust stable, that is the equilibrium of system (2.5) is global robust stable.

Remark 1. The matrix $\Pi$ in (4.3) can't be represented in actual manipulation because the elements of the matrix is varying with the uncertainty. In our proof, by the convexity of $\Theta, \Upsilon$ and $\Omega$, we overcome this problem and get the condition (4.2) for global robust stability of system (2.5).

Remark 2. In the above theorem, if $D_{\theta}=D, A_{v}=A$ and $B_{\omega}=B$, we get the stability condition for the nominal system. 


\subsection{Delay-dependent robust stability}

In this section we discuss the problem of delay-dependent robust stability for system (2.5). We give the stable condition using Leibniz-Newton formula and free weighting matrix.

Theorem 4.3. Suppose that $A(1)$ and $A(2)$ are satisfied, if there exist $P=$ $P^{\mathrm{T}}>0, Q=Q^{\mathrm{T}}>0, R=R^{\mathrm{T}}>0, \wedge=\operatorname{diag}\left(\lambda_{1}, \lambda_{2}, \ldots, \lambda_{n}\right) \geq 0$,

$$
X=\left[\begin{array}{llll}
X_{11} & X_{12} & X_{13} & X_{14} \\
X_{12}^{\mathrm{T}} & X_{22} & X_{23} & X_{24} \\
X_{13}^{\mathrm{T}} & X_{23}^{\mathrm{T}} & X_{33} & X_{34} \\
X_{14}^{\mathrm{T}} & X_{24}^{\mathrm{T}} & X_{34}^{\mathrm{T}} & X_{44}
\end{array}\right] \geq 0
$$

and some free matrixes $N_{i}(i=1,2,3,4)$ such that

$$
\bar{\Gamma}=\left[\begin{array}{cccc}
\bar{\Gamma}_{11} & \bar{\Gamma}_{12} & \bar{\Gamma}_{13} & \bar{\Gamma}_{14} \\
\bar{\Gamma}_{12}^{\mathrm{T}} & \bar{\Gamma}_{22} & \bar{\Gamma}_{23} & \bar{\Gamma}_{24} \\
\bar{\Gamma}_{13}^{\mathrm{T}} & \bar{\Gamma}_{23}^{\mathrm{T}} & \bar{\Gamma}_{33} & \bar{\Gamma}_{34} \\
\bar{\Gamma}_{14}^{\mathrm{T}} & \bar{\Gamma}_{24}^{\mathrm{T}} & \bar{\Gamma}_{34}^{\mathrm{T}} & \bar{\Gamma}_{44}
\end{array}\right]<0
$$

and

$$
\bar{\Pi}=\left[\begin{array}{ccccc}
X_{11} & X_{12} & X_{13} & X_{14} & N_{1} \\
X_{12}^{\mathrm{T}} & X_{22} & X_{23} & X_{24} & N_{2} \\
X_{13}^{\mathrm{T}} & X_{23}^{\mathrm{T}} & X_{33} & X_{34} & N_{3} \\
X_{14}^{\mathrm{T}} & X_{24}^{\mathrm{T}} & X_{34}^{\mathrm{T}} & X_{44} & N_{4} \\
N_{1}^{\mathrm{T}} & N_{2}^{\mathrm{T}} & N_{3}^{\mathrm{T}} & N_{4}^{\mathrm{T}} & R
\end{array}\right]>0
$$

hold, where

$$
\begin{aligned}
& \bar{\Gamma}_{11}=-P \bar{D}_{\theta}-\bar{D}_{\theta}^{\mathrm{T}} P+\tau \bar{D}_{\theta}^{\mathrm{T}} R \bar{D}_{\theta}+N_{1}+N_{1}^{\mathrm{T}}+\tau X_{11} \\
& \bar{\Gamma}_{12}=N_{2}^{\mathrm{T}}-N_{1}+\tau X_{12}, \\
& \bar{\Gamma}_{13}=P \bar{A}_{v}-\bar{D}_{\theta}^{\mathrm{T}} \wedge-\tau \bar{D}_{\theta}^{\mathrm{T}} R \bar{A}_{v}+N_{3}^{\mathrm{T}}+\tau X_{13} \\
& \bar{\Gamma}_{14}=P \bar{B}_{\omega}-\tau \bar{D}_{\theta}^{\mathrm{T}} R \bar{B}_{\omega}+N_{4}^{\mathrm{T}}+\tau X_{14} \\
& \bar{\Gamma}_{22}=-N_{2}^{\mathrm{T}}-N_{2}+\tau X_{22} \\
& \bar{\Gamma}_{23}=-N_{3}^{\mathrm{T}}+\tau X_{23} \\
& \bar{\Gamma}_{24}=-N_{4}^{\mathrm{T}}+\tau X_{24} \\
& \bar{\Gamma}_{33}=\wedge \bar{B}_{\omega}+\bar{B}_{\omega}^{\mathrm{T}} \wedge+\tau \bar{A}_{v}^{\mathrm{T}} R \bar{A}_{v}+\tau X_{33} \\
& \bar{\Gamma}_{34}=\wedge \bar{B}_{\omega}+\tau \bar{A}_{v}^{\mathrm{T}} R \bar{B}_{\omega}+\tau X_{34} \\
& \bar{\Gamma}_{44}=\tau \bar{B}_{\omega}^{\mathrm{T}} R \bar{B}_{\omega}-Q+\tau X_{44}
\end{aligned}
$$

then system (2.5) is robust stable. 
Proof. Choose a Lyapunov function candidate

$$
\begin{aligned}
V_{[z, \kappa]}= & z^{\mathrm{T}}(t) P z(t)+2 \sum_{i=1}^{n} \lambda_{i} \int_{0}^{z_{i}(t)} f_{i}(s) \mathrm{d} s \\
& +\int_{t-\tau}^{t} \kappa^{\mathrm{T}}(\rho) Q \kappa(\rho) \mathrm{d} \rho+\int_{-\tau}^{0} \int_{t+\theta}^{t} \dot{z}^{\mathrm{T}}(s) R z(s) \mathrm{d} s \mathrm{~d} \theta
\end{aligned}
$$

where $P=P^{\mathrm{T}}>0, Q=Q^{\mathrm{T}}>0, R=R^{\mathrm{T}} \geq 0$ and $\lambda_{i} \geq 0(i=1,2, \ldots, n)$ need to be determined. Using the Leibniz-Newton formula yields

$$
z(t)-z(t-\tau)-\int_{t-\tau}^{t} \dot{z}(s) \mathrm{d} s=0 .
$$

Then for any constant matrixes $N_{i}(i=1,2,3,4)$ with appropriate dimensions, the following equation is true.

$$
\begin{array}{r}
2\left[z(t)^{\mathrm{T}} N_{1}+z(t-\tau)^{\mathrm{T}} N_{2}+\kappa(t)^{\mathrm{T}} N_{3}+\kappa(t-\tau)^{\mathrm{T}} N_{4}\right] \\
\cdot\left[z(t)-z(t-\tau)-\int_{t-\tau}^{t} \dot{z}(s) \mathrm{d} s\right]=0 .
\end{array}
$$

On the other hand, for any constant matrix $X$ with appropriate dimensions, the following equation is also true.

$$
\left[\begin{array}{c}
z(t) \\
z(t-\tau) \\
\kappa(t) \\
\kappa(t-\tau)
\end{array}\right]^{\mathrm{T}}\left[\begin{array}{cccc}
\tau\left(X_{11}-X_{11}\right) & \tau\left(X_{12}-X_{12}\right) & \tau\left(X_{13}-X_{13}\right) & \tau\left(X_{14}-X_{14}\right) \\
\tau\left(X_{12}-X_{12}\right)^{T} & \tau\left(X_{22}-X_{22}\right) & \tau\left(X_{23}-X_{23}\right) & \tau\left(X_{24}-X_{24}\right) \\
\tau\left(X_{13}-X_{13}\right)^{T} & \tau\left(X_{23}-X_{23}\right)^{T} & \tau\left(X_{33}-X_{33}\right) & \tau\left(X_{34}-X_{34}\right) \\
\tau\left(X_{14}-X_{14}\right)^{T} & \tau\left(X_{24}-X_{24}\right)^{T} & \tau\left(X_{34}-X_{34}\right)^{T} & \tau\left(X_{44}-X_{44}\right)
\end{array}\right]\left[\begin{array}{c}
z(t) \\
z(t-\tau) \\
\kappa(t) \\
\kappa(t-\tau)
\end{array}\right]=0 .
$$

Differentiating $V_{[z, \kappa]}$ along the solution of system (4.1) by the chain role and adding the terms on the left side of (4.10) and (4.11) to (4.12) yields

$$
\left.\dot{V}_{[z, \kappa]}\right|_{(4.1)}=\xi^{\mathrm{T}}(t) \Gamma \xi(t)-\int_{t-\tau}^{t} \zeta(t, s)^{\mathrm{T}} \Pi \zeta(t, s) \mathrm{d} s
$$

where

$$
\xi(t)=\left[z(t)^{\mathrm{T}}, z^{\mathrm{T}}(t-\tau), \kappa^{\mathrm{T}}(t), \kappa^{\mathrm{T}}(t-\tau)\right]^{\mathrm{T}}, \zeta(t, s)=\left[\xi^{\mathrm{T}}(t), \dot{z}^{\mathrm{T}}(t)\right]^{\mathrm{T}} .
$$

From the definition of set $\Theta, \Upsilon$ and $\Omega$, we know $\Theta, \Upsilon$ and $\Omega$ are convex sets. By the convexity of $\Theta, \Upsilon$ and $\Omega$, we obtain that if $\bar{\Gamma}<0$ and $\bar{\Pi}$ hold for every $\bar{D}_{\theta}, \bar{A}_{v}$ and $\bar{B}_{\omega}$, we have

$$
\Gamma=\left[\begin{array}{cccc}
\Gamma_{11} & \Gamma_{12} & \Gamma_{13} & \Gamma_{14} \\
\Gamma_{12}^{T} & \Gamma_{22} & \Gamma_{23} & \Gamma_{24} \\
\Gamma_{13}^{T} & \Gamma_{23}^{T} & \Gamma_{33} & \Gamma_{34} \\
\Gamma_{14}^{T} & \Gamma_{24}^{T} & \Gamma_{34}^{T} & \Gamma_{44}
\end{array}\right]<0,
$$


where

$$
\begin{aligned}
& \Gamma_{11}=-P D_{\theta}-D_{\theta}^{\mathrm{T}} P+\tau D_{\theta}^{\mathrm{T}} R D_{\theta}+N_{1}+N_{1}^{\mathrm{T}}+\tau X_{11}, \\
& \Gamma_{12}=N_{2}^{\mathrm{T}}-N_{1}+\tau X_{12}, \\
& \Gamma_{13}=P A_{v}-D_{\theta}^{\mathrm{T}} \wedge-\tau D_{\theta}^{\mathrm{T}} R A_{v}+N_{3}^{\mathrm{T}}+\tau X_{13}, \\
& \Gamma_{14}=P B_{\omega}-\tau D_{\theta}^{\mathrm{T}} R B_{\omega}+N_{4}^{\mathrm{T}}+\tau X_{14}, \\
& \Gamma_{22}=-N_{2}^{\mathrm{T}}-N_{2}+\tau X_{22}, \\
& \Gamma_{23}=-N_{3}^{\mathrm{T}}+\tau X_{23}, \\
& \Gamma_{24}=-N_{4}^{\mathrm{T}}+\tau X_{24}, \\
& \Gamma_{33}=\wedge B_{\omega}+B_{\omega}^{\mathrm{T}} \wedge+\tau A_{v}^{\mathrm{T}} R A_{v}+\tau X_{33}, \\
& \Gamma_{34}=\wedge B_{\omega}+\tau A_{v}^{\mathrm{T}} R B_{\omega}+\tau X_{34}, \\
& \Gamma_{44}=\tau B_{\omega}^{\mathrm{T}} R B_{\omega}-Q+\tau X_{44} .
\end{aligned}
$$

Therefore we get $\left.\dot{V}_{[z, \kappa]}\right|_{(4.1)}<0$. From the definition of absolute stability of system, it follows that system (4.1) is global robust stable, which means the equilibrium of system (2.5) is global robust stable.

Remark 3. The matrix $\Gamma$ and $\Pi$ in (4.12) can't be represented in actual manipulation because the elements of the matrixes are varying with the uncertainty. In our proof, by the convexity of $\Theta, \Upsilon$ and $\Omega$, we overcome this problem and get the conditions (4.6) and (4.7) for global robust stability of system (2.5).

Remark 4. In the above theorem, if $D_{\theta}=D, A_{v}=A$ and $B_{\omega}=B$, we get the time-dependent stability condition for the nominal system.

\section{Numerical simulations}

In this section, we present a numerical example to illustrate the proposed stability criterion, which shows our theorems are effective.

Example. Consider a delayed two-neurons network (2.5) with the uncertain parameter matrixes given by $D_{\theta}=D+\sum_{i=1}^{k} \theta_{i} D_{i}, A_{v}=A+\sum_{j=1}^{p} v_{j} A_{j}$, $B_{\omega}=B+\sum_{l=1}^{m} \omega_{l} B_{l}$, where

$$
\begin{gathered}
D=\left[\begin{array}{ll}
2 & 0 \\
1 & 1
\end{array}\right], D_{1}=\left[\begin{array}{ll}
1 & 0 \\
0 & 1
\end{array}\right], D_{2}=\left[\begin{array}{ll}
0 & 1 \\
1 & 0
\end{array}\right], \\
A=\left[\begin{array}{cc}
-2 & -0.2 \\
-0.2 & -2.8
\end{array}\right], A_{1}=\left[\begin{array}{ll}
1 & 0 \\
0 & 1
\end{array}\right], A_{2}=\left[\begin{array}{ll}
0 & 1 \\
1 & 0
\end{array}\right], \\
B=\left[\begin{array}{cc}
0.5 & -0.9 \\
-0.5 & -0.8
\end{array}\right], B_{1}=\left[\begin{array}{ll}
1 & 0 \\
0 & 1
\end{array}\right], B_{2}=\left[\begin{array}{ll}
0 & 1 \\
1 & 0
\end{array}\right],
\end{gathered}
$$

and $\theta_{1} \in[-0.2,0.2], \theta_{2} \in[-0.5,0.5], v_{1} \in[-0.3,0.3], v_{2} \in[-0.1,0.1], \omega_{1} \in$ $[-0.2,0.2], \omega_{2} \in[-0.3,0.3]$. The discontinuous function in the system is defined as follows

$$
g(x)= \begin{cases}1+x, & x>0 \\ 0, & x=0 \\ -1+x, & x<0\end{cases}
$$




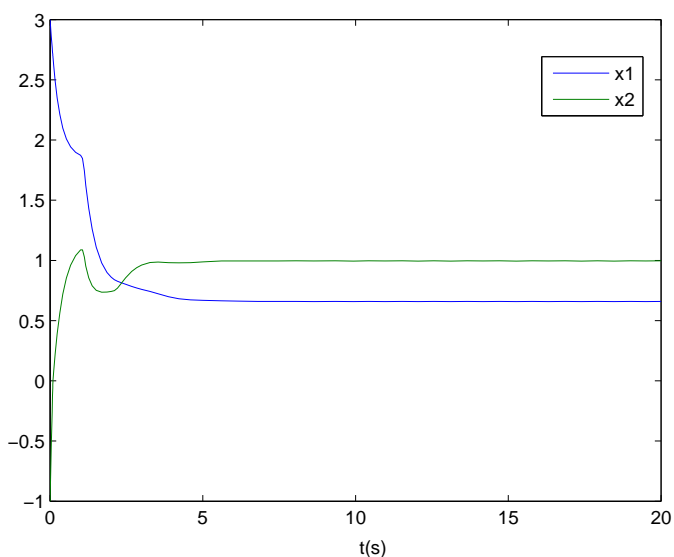

Fig. 1: Dynamical behaviors of the states $x_{1}(t)$ and $x_{2}(t)$ in Example 1 for $t \in[0,20]$.

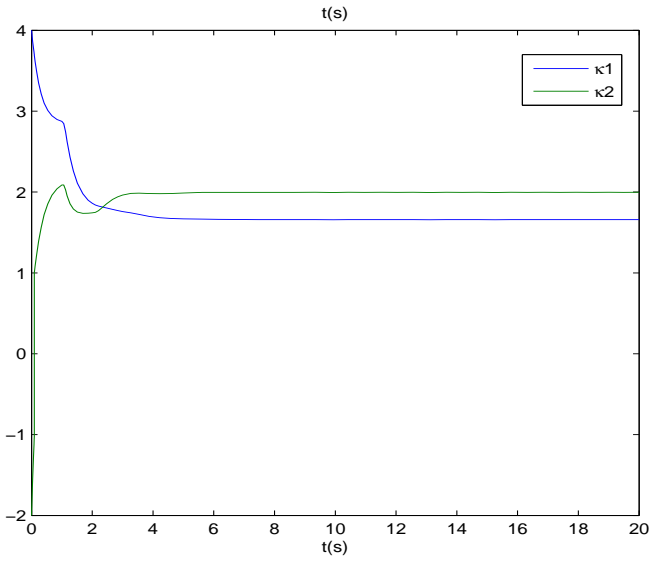

Fig. 2: Dynamical behaviors of the states $\kappa_{1}(t)$ and $\kappa_{2}(t)$ in Example 1 for $t \in[0,20]$.

Using Matlab LMI boolbox, it turns out the uncertain parameter matrixes satisfies all the conditions in the Theorem 4.2. As a special case, we choose the system as follows;

$$
D=\left[\begin{array}{ll}
2 & 0 \\
1 & 1
\end{array}\right], A=\left[\begin{array}{cc}
-2 & -0.2 \\
-0.2 & -2.8
\end{array}\right], B=\left[\begin{array}{cc}
0.5 & -0.9 \\
-0.5 & -0.8
\end{array}\right]
$$


Given the initial condition $x(\phi)=[3-1]^{\mathrm{T}}, \theta \in[-1,0], I=[6,10]^{T}$. The trajectories of $x_{1}(t)$ and $x_{2}(t)$ are shown in Fig.1, and the trajectories of output, $\kappa_{1}(t)$ and $\kappa_{2}(t)$ are shown in Fig.2.

\section{Conclusion}

In this brief, we have a research of the robust stability problem for time delay discontinuous system, in which the self-connection matrix is a positive matrix. Sufficient conditions have been obtained for the delay-independent robust stability and time-dependent robust stability in term of linear matrix inequalities based on Lyapunov function as well as Filippov theoria. Some free weighting matrices that express the influence of $z(t-\tau)$ and $z(t)-\int_{t-\tau}^{t} \dot{z}(s) d s$ are determined based on linear matrix inequalities which makes it easy to choose suitable ones. Numerical example shows the effectiveness of our results.

\section{References}

[1] J. Aubin and A. Cellina, Differential Inclusions, Springer-Verlag, Berlin, 1984.

[2] G. Avitabile, M. Forti, S. Manetti, and M. Marini, On a class of nonsymmetrical neural networks with application to ADC, IEEE Trans. Circuits Syst. 38 (1991), 202-209.

[3] S. Boyd, L. Ghaoui, E. Feron, and V. Balakrishnan, Linear matrix inequalities in system and control theory, SIAM Studies in Applied Mathematics, 15. Society for Industrial and Applied Mathematics (SIAM), Philadelphia, PA, 1994.

[4] A. Filippov, Differential Equations with Discontinuous Righthand Sides, Mathematics and its Applications (Soviet Series), 18. Kluwer Academic Publishers Group, Dordrecht, 1988.

[5] M. Forti, M. Grazzini, P. Nistri, and L. Pancioni, Generalized lyapunov approach for convergence of neural networks with discontinuous or non-lipschitz activations, Physica D: Nonlinear Phenomena 214 (2006), 88-99.

[6] M. Forti, S. Manetti, and M. Marini, A condition for global convergence of a class of symmetric neural networks, IEEE Trans. Circuits Syst. I 39 (1992), 480-483.

[7] M. Forti and P. Nistri, Global convergence of neural networks with discontinuous neuron activations, IEEE Trans. Circuits Syst. I 50 (2003), no. 11, 1421-1435.

[8] M. Forti, P. Nistri, and D. Papini, Global exponential stability and global convergence in finite time of delayed neural networks with infinite gain, IEEE Trans. Neural Netw. 16 (2005), no. 6, 1449-1463.

[9] S. Grossberg, Nonlinear neural networks: Principles, mechanisms and architectures, Neural Netw. 1 (1988), 17-61.

[10] Y. He, M. Wu, and J. She, Parameter-dependent Lyapunov functional for stability of the time-delay system with polytopic-type uncertainty, IEEE Trans. Automatic Control 49 (2004), 828-832.

[11] M. Hirsch, Convergent activation dynamics in continuous time networks, Neural Netw. 2 (1989), no. 5, 331-349.

[12] S. Hu and J. Wang, Global stability of a class of continuous-time recurrent neural networks, IEEE Trans. Circuits Systems I Fund. Theory Appl. 49 (2002), no. 9, 13341347.

[13] X. Liang and J. Wang, A recurrent neural network for nonlinear optimization with a continuously differentiable objective function and bound constraints, IEEE Trans. Neural Netw. 11 (2000), no. 6, 1251-1262. 
[14] C. Lim, Y. Park, and S. Moon, Robust saturation controller for linear time-invariant system with structured real parameter uncertainties, J. Sound Vibra. 294 (2006), no. $1-2,1-14$.

[15] W. Lu and T. Chen, Dynamical behaviors of delayed neural network systems with discontinuous activation functions, Neural Computation 18 (2006), 683-708.

[16] Y. Moon, P. Park, W. Kwon, and Y. Lee, Delay-dependent robust stabilization of uncertain state-delayed systems, Internat. J. Control $\mathbf{7 4}$ (2001), no. 14, 1447-1455.

[17] S. Niculescu, Delay Effects on Stability: A Robust Control Approach, Lecture Notes in Control and Information Sciences, 269. Springer-Verlag London, Ltd., London, 2001.

[18] D. Papini and V. Taddei, Global exponential stability of the periodic solution of a delayed neural network with discontinuous activations, Phys. Lett. A 343 (2005), 117-128.

[19] P. Park, A delay-dependent stability criterion for systems with uncertain time-invariant delays, IEEE Trans. Automat. Control 44 (1999), no. 4, 876-877.

ZENGYUN WANG

College of Mathematics and ECONOMtrics

HUNAN UNIVERSITY

Changsha, 410082, P. R. China

E-mail address: shunshuang1953@163.com

LIHONG HUANG

College of Mathematics and Economtrics

HUNAN UNIVERSITY

Changsha, 410082, P. R. China

E-mail address: 1hhuang@hnu.cn

Yi ZUO

School of Energy and Power Engineering

Changsha University of Science And Technology

Changsha, 410004

AND

Department of Applied Mathematics

UNIVERSITY OF WATERLOO

Waterloo, Ontario N2L 3G1, Canada

E-mail address: yizuohnu@gmail.com

LiNGLiNG ZHANG

College of Mathematics and Economtrics

HunAN UNIVERSITY

Changsha, 410082, P. R. China 